\title{
Existence of Solutions for Some $p(x)$-polyharmonic Elliptic Kirchhoff Equations
}

\section{Yijian Ge}

School of Science, University of Shanghai for Science and Technology, Shanghai, China

Email: yjGeusst@163.com

How to cite this paper: Ge, Y.J. (2019) Existence of Solutions for Some $p(x)$-polyharmonic Elliptic Kirchhoff Equations. $A d$ vances in Pure Mathematics, 9, 863-878. https://doi.org/10.4236/apm.2019.910043

Received: September 19, 2019

Accepted: October 18, 2019

Published: October 21, 2019

Copyright (C) 2019 by author(s) and Scientific Research Publishing Inc. This work is licensed under the Creative Commons Attribution International License (CC BY 4.0).

http://creativecommons.org/licenses/by/4.0/

\begin{abstract}
In this paper, we study the existence of solution for some $p(x)$-polyharmonic Kirchhoff equations. The latter is allowed to vanish at the origin (degenerate case). Firstly, we study the existence of solutions of approximate equations. Secondly, we prove the existence of the solutions of the original equation. The main tool is the Schauder's Theorem.
\end{abstract}

\section{Keywords}

$p(x)$-polyharmonic Kirchhoff Equations, Existence of Solutions,

Schauder's Fixed Point Theorem

\section{Introduction}

In this paper, we prove the existence of solution of Dirchlet problems involving the $p$-polyharmonic operators $\Delta_{p}^{s}$. We consider

$$
\begin{cases}M\left(\|u\|^{p}\right) \Delta_{p}^{s} u+a(x) g(u)=f(x) & \text { in } \Omega, \\ \left.D^{\alpha} u(x)\right|_{\partial \Omega}=0 & \forall \alpha, \text { with }|\alpha| \leq s-1,\end{cases}
$$

where $\Omega \subset \mathbb{R}^{N}$ is a bounded domain, $p \geq 2, s=1,2, \cdots,\|\cdot\|$ is denoted in section 2, and $f(x) \in L^{1}(\Omega), 0 \leq a(x) \in L^{1}(\Omega)$. Here, the $p$-polyharmonic operator is defined by

$$
\Delta_{p}^{s} u=\left\{\begin{array}{ll}
-\operatorname{div} \Delta^{j-1}\left(\left|D \Delta^{j-1} u\right|^{p-2}\right) D \Delta^{j-1} u, & s=2 j-1, \\
\Delta^{j}\left(\left|\Delta^{j} u\right|^{p-2} \Delta^{j} u\right), & s=2 j,
\end{array} \quad j=1,2, \cdots,\right.
$$

which becomes the usual $p$-Laplacian for $s=1$. Kratochvl and Necâs introduced the $p$-biharmonic operator in [1] [2] [3] to study the physical equations, the $p$-biharmonic operator for $s=2$ and the polyharmonic operator for 
$p=2$, which reduces to the more appoximate case

$$
\left\{\begin{array}{ll}
M\left(\|u\|^{2}\right)(-\Delta u)^{s}=f(x, u) & \text { in } \Omega, \\
\left.D^{\alpha} u(x)\right|_{\partial \Omega}=0 & \forall \alpha, \text { with }|\alpha| \leq s-1
\end{array} .\right.
$$

We introduce for $s=1,2, \cdots$, the main $s$-order differential operator

$$
\mathcal{D}_{s} u=\left\{\begin{array}{ll}
D \Delta^{j-1} u & \text { if } s=2 j-1, \\
\Delta^{j} u & \text { if } s=2 j
\end{array} \quad j=1,2, \cdots .\right.
$$

Note that $\mathcal{D}_{s}$ is an $n$-vectorial operator when $s$ is odd and $n>1$, while it is a scalar operator when $s$ is even.

In our hypothesis, the Kirchhoff function $M: R_{0}^{+} \rightarrow R_{0}^{+}$is assumed to be continuous and to verify the structural assumptions $(M)$ :

$\left(\mathrm{M}_{1}\right) M$ is non-decreasing;

$\left(\mathrm{M}_{2}\right)$ there exists a number $\gamma \in\left[1, p_{s}\right)$ such that for all $t \in R_{0}^{+}$;

$$
t M(t) \leq \gamma \hat{M}(t), \quad \text { where } \hat{M}(t)=\int_{0}^{t} M(\theta) \mathrm{d} \theta ;
$$

$\left(\mathrm{M}_{3}\right)$ for all $t \geq \sigma$, there exists $m_{0}=m_{0}(\sigma)>0$ such that $M(t) \geq m_{0}$ for all $\sigma \geq 0$

We introduce the Sobolev critical exponent $p_{s}^{*}$ and the number $p_{s}$ defined by following

$$
p_{s}^{*}=\left\{\begin{array}{ll}
\frac{n p}{n-s p} & \text { if } n>s p, \\
\infty & \text { if } n \leq s p .
\end{array} \quad p_{s}=\frac{p_{s}^{*}}{p}= \begin{cases}\frac{n}{n-s p} & \text { if } n>s p, \\
\infty & \text { if } n \leq s p .\end{cases}\right.
$$

A very special Kirchhoff function verifying $(M)$ is denoted by

$$
M(t)=a+b \gamma t^{\gamma-1}, a, b \geq 0, a+b>0, \gamma \begin{cases}\in\left(1, p_{s}\right) & \text { if } b>0, \\ =1 & \text { if } b=0 .\end{cases}
$$

when $M$ is of the type (6) and $a>0, b \geq 0$, problem (1) is said to be non-degenerate, while it is called degenerate if $a=0$. Besides, problem (2) reduces to the usual well-known quasilinear elliptic equation while $a>0, b=0$. The existence of positive solutions of non-degenerate Kirchhoff-type problems has been proved in [4] [5] for $L=1$. The novelty of this paper is to treat the degenerate case with allowing Kirchhoff function to take the zero value. Several authors have considered fourth order problems with nonlinear boundary conditions involving third order derivatives, see [6]. The classical counterpart of our problem models containning several interesting phenomena were deeply studied in physicals even in the one-dimensional case. It dates back to 1883 when Kirchhoff proposed his celebrated equation:

$$
\rho \frac{\partial^{2} u}{\partial t^{2}}-\left(\frac{P_{0}}{h}+\frac{E}{2 L} \int_{0}^{L}\left|\frac{\partial u}{\partial x}\right|^{2} \mathrm{~d} x\right) \frac{\partial^{2} u}{\partial x^{2}}=0,
$$

as a nonlinear extension of D'Alambert's wave equation for free vibrations for elastic strings. 
Here we study a stationary version of Kirchhoff-type problems, where $u=u(x)$ is the lateral displacement at the space coordinate $\chi$ and $M$ is typically a line with positive slope. Our result allows $M$ to have this property. The classical Kirchhoff theory described further details and physical models, which can be found in [7] [8]. In the standard case $L=2$, problem of type (2) arise in the theory of bending extensible elastic beams. There $u=u(x)$ denotes a thin extensible elastic beam. The function $f$ models a small changes with effect in the length of beam but acts as a force exerted on the beam. We read to [6] and the references therein for a discussion about modelling of Kirchhoff-type strings and beams. We cite the wide literature on the subject, the works [9] [10] [11] [12], where Kirchhoff-type problems new studied by exploiting different methods.

We recall that study of semilinear case with datum $f(x) \in L^{1}(\Omega)$ in [13] [14] [15] [16], with respect to (1), we assume that the coefficient $a(x)$ of the zero order term and to the datum $f(x)$, in addition to imposing that

$$
f(x), a(x) \in L^{1}(\Omega) \text {, }
$$

and there exists $Q>0$ such that, for $x \in \Omega$ a.e.,

$$
|f(x)| \leq Q a(x) \text {. }
$$

There is assumption that $g(s)$ is continuous function satisfies

$$
\lim _{s \rightarrow-\infty} g(s)=-\infty \text { and } \lim _{s \rightarrow+\infty} g(s)=\infty .
$$

There has been an increasing interest in studying equations involving $p(x)$-Laplace operators over the last few decades. Motivated by theoretical research in the regularizing effect of the interaction between the coefficient of the zero order term and the datum $f(x) \in L^{1}(\Omega)$ in some nonlinear Dirchlet problems, we pay attention to the existence of solutions for $p(x)$-polyharmonic Kirchhoff equations. Now we consider the problems

$$
\begin{cases}M(\varphi(u)) \Delta_{p(x)}^{s} u+a(x) g(u)=f(x) & \text { in } \Omega, \\ \left.D^{\alpha} u(x)\right|_{\partial \Omega}=0 & \forall \alpha, \text { with }|\alpha| \leq s-1,\end{cases}
$$

where $\Omega \subset \mathbb{R}$ is a bounded domain Lipschitz boundary, $M$ is a degenerate Kirchhoff function and $p \in C(\bar{\Omega})$. More details and conditions are given in section 4 . The $p(x)$-polyharmonic operator is given by

$$
\Delta_{p(x)}^{s} u=\left\{\begin{array}{ll}
-\operatorname{div} \Delta^{j-1}\left(\left|D \Delta^{j-1} u\right|^{p(x)-2}\right) D \Delta^{j-1} u, & s=2 j-1, \\
\Delta^{j}\left(\left|\Delta^{j} u\right|^{p(x)-2} \Delta^{j} u\right), & s=2 j,
\end{array} \quad j=1,2, \cdots .\right.
$$

The author exploits the symmetric mountain pass theorem to proves the multiplicity of solutions for $p(x)$-polyharmonic elliptic Kirchhoff equations in [17]. In contrast, in this paper, the keystone of the proofs them is the deduction, by condition (7), (8), of the $L^{\infty}$-estimate of the approximated solutions, we prove the problem existing a solution $u \in W_{0}^{s \cdot p(\cdot)}(\Omega) \cap L^{\infty}(\Omega)$.

This paper is organized as follows. In Section 2, we introduce some basic no- 
tation and properties in variable exponent Sobolev spaces. In Section 3, we prove the problem (1) ( $p \equiv$ Const ) existing a solution $u \in W_{0}^{s . p}(\Omega) \cap L^{\infty}(\Omega)$. In Section 4 , we treat the more delicate case $p=p(x)$.

\section{Notations and Preliminaries}

In this section, we briefly introduce some basic results and notations. Let $\Omega$ be a bounded domain in $\mathbb{R}^{N}$, we denote a multi-index $\alpha=\left(\alpha_{1}, \alpha_{2}, \cdots, \alpha_{n}\right) \in \mathbb{N}_{0}^{n}$, with length $|\alpha|=\sum_{i=1}^{n} \alpha_{i} \leq s$, such that the corresponding partial differentation:

$$
D^{\alpha}=\frac{\partial^{|\alpha|}}{\partial x_{1}^{\alpha_{1}} \partial x_{2}^{\alpha_{2}} \cdots \partial x_{n}^{\alpha_{n}}} .
$$

Write:

$$
\|u\|_{W^{s, p}(\Omega)}=\left(\sum_{|\alpha| \leq s}\left\|D^{\alpha} u\right\|^{p}\right)^{\frac{1}{p}},
$$

where $\|\cdot\|_{p}$ denotes the standard $L^{p}$-norm. See [18], we denote the space $W_{0}^{s, p}(\Omega)$ is the completion of $C_{0}^{\infty}(\Omega)$ with respect to the standard norm of $W^{s, p}(\Omega)$. Moreover, denote $\mathcal{D}^{s, p}(\Omega)$ be the completion of $C_{0}^{\infty}(\Omega)$, with respect to the norm:

$$
\|u\|_{\mathcal{D}^{s, p}(\Omega)}=\left(\sum_{|\alpha|=s}\left\|D^{\alpha} u\right\|^{p}\right)^{\frac{1}{p}} .
$$

By the poncare inequality, there exists a positive constant $\mathcal{K}=\mathcal{K}(n, p, \Omega)$, with $m=s, p \geq 1$, such that

$$
\|u\|_{W^{s, p}(\Omega)} \leq \mathcal{K}\|u\|_{D^{s, p}(\Omega)}, \forall u \in W_{0}^{s, p}(\Omega)
$$

Hence, we obtain that the norms $\|\cdot\|_{W^{s, p}(\Omega)}$ are equivalent, so that the two completions of $C_{0}^{\infty}(\Omega)$, with corresponding these norms, namely

$$
W_{0}^{s, p}(\Omega)=\mathcal{D}^{s, p}(\Omega) .
$$

We endow the vectorial space $\left[L^{p}(\Omega)\right]^{n}$, with respect to the norm

$$
\|v\|_{p}=\left(\sum_{i=1}^{n}\left\|\beta_{i}\right\|_{p}^{p}\right)^{\frac{1}{p}},
$$

where $v=\left(\beta_{1}, \beta_{2}, \cdots, \beta_{n}\right)$ and $n>1$, we still use the same symbol $\|\cdot\|_{p}$ to denote both the standard $L^{p}$-norm in the scalar space $L^{p}(\Omega)$ and the norm define in (15), in the vectorial space $\left[L^{p}(\Omega)\right]^{n}$.

For $s=2,1<p<\infty$, by the Caldéron-Zygmund inequality, see details in [19] [20], there exists a constant $k_{2}=k_{2}(n, p)>0$ such that:

$$
\|u\|_{\mathcal{D}^{2, p}(\Omega)} \leq k_{2}\left\|\mathcal{D}_{2} u\right\|_{p}, \forall u \in W_{0}^{2, p}(\Omega) .
$$

Proposition 2.1. If $p \in(1, \infty)$ and $s=1,2, \cdots$, then there exists a positive constant $k_{s}=k_{s}(n, p)$ such that:

$$
\|u\|_{\mathcal{D}^{s, p}(\Omega)} \leq k_{s}\left\|\mathcal{D}_{s} u\right\|_{p}, \quad \forall u \in W_{0}^{s, p}(\Omega) .
$$


where $\mathcal{D}_{s}$ is denoted in (4), see also in [17].

Hence, from now on we endow $W_{0}^{s, p}(\Omega)$ with the norm $\|\cdot\|=\left\|\mathcal{D}_{s} \cdot\right\|_{p}$, which is equivalent to the standard Sobolev norm.

Remark 2.1. For all $s=1,2, \cdots, 1<p<\infty . W_{0}^{s, p}(\Omega)$ is a separable, uniformly convex, reflexive, real Banach space.

Note that, when $p=2$, this norm is introduced by the inner product

$$
\langle u, v\rangle=\int_{\Omega} \mathcal{D}_{s} u \mathcal{D}_{s} v \mathrm{~d} x, \forall u, v \in H_{0}^{s}(\Omega)
$$

when $s$ is even the operation between $\mathcal{D}_{s} u$ and $\mathcal{D}_{s} v$ is scalar multiplication, while $s$ is odd, it is the $n$-Euclidean scalar product.

Lemma 2.1. See [21] (Schuader's theorem) Let $F$ be a completely continuous map and let $K$ be a convex, bounded, closed and invariant subset of $X$. Then $F$ has a fixed point in $K$.

$F$ is completely continuous map:

1) $F$ is continuous.

2) For every $B$ is bounded subset of $X$, then $\overline{F(B)}$ is compact.

Proposition 2.2. See [18], for $1 \leq h<p_{s}{ }^{*}$, the embedding $W_{0}^{s, p}(\Omega), L^{h}(\Omega)$ is compact and continuous, there exists $\delta_{h}=\delta_{h}(n, p, s, \Omega)>0$, such that.

$$
\|u\|_{h} \leq \delta_{h}\|u\|, \forall u \in W_{0}^{s, p}(\Omega) .
$$

We study problem (1) for a solution, we understand:

$$
\left\{\begin{array}{l}
u \in W_{0}^{s, p}(\Omega) \cap L^{\infty}(\Omega), \\
M\left(\|u\|^{p}\right) \int_{\Omega}\left|\mathcal{D}_{s} u\right|^{p-2} \mathcal{D}_{s} u \mathcal{D}_{s} v \mathrm{~d} x+\int_{\Omega} a(x) g(u) \varphi=\int_{\Omega} f(x) \varphi, \\
\varphi \in W_{0}^{s, p}(\Omega) \cap L^{\infty}(\Omega) .
\end{array}\right.
$$

where $\mathcal{D}_{s}$ is the operator in (4) and $\int_{\Omega}\left|\mathcal{D}_{s} u\right|^{p-2} \mathcal{D}_{s} u \mathcal{D}_{s} v \varphi \mathrm{d} x$ is the p-polyharmonic operator $\Delta_{p}^{s}$ in weak sense.

\section{Existence and Uniqueness of Solution for (1)}

In order to study the solution of problem (1), we consider problems:

$$
\begin{cases}M\left(\left\|u_{n}\right\|^{p}\right) \Delta_{p}^{s} u_{n}+a_{n}(x) g\left(u_{n}\right)=f_{n} & \text { in } \Omega, \\ \left.D^{\alpha} u_{n}(x)\right|_{\partial \Omega}=0 & \forall \alpha, \text { with }|\alpha| \leq s-1 .\end{cases}
$$

where $\Omega$ is a bounded domain in $\mathbb{R}^{N}, p \in[2, \infty)$, and $s=1,2, \cdots$. Indeed, suppose $f(x) \in L^{1}(\Omega), \quad 0 \leq a(x) \leq L^{1}(\Omega)$, and exist $h(x) \in L^{q^{\prime}}(\Omega)$, then $|g(s)| \leq h(x)$.

Let us define:

$$
a_{n}(x)=\frac{a(x)}{1+\frac{Q}{n}|a(x)|}, \quad f_{n}(x)=\frac{f(x)}{1+\frac{1}{n}|f(x)|} .
$$

and that we choose $k_{0}>0$, such that

$$
g(t) t \geq 0,|g(t)| \geq Q .
$$


for every $t \geq k_{0}$.

Theorem 3.1. There is a solution $u_{n} \in W_{0}^{s, p}(\Omega)$ to the problem (21).

Proof. Since $\varphi=s\left(1+\frac{s}{n}\right)^{-1}$ is increasing, we deduced by (8) that,

$$
\left|f_{n}(x)\right|=\frac{|f(x)|}{1+\frac{1}{n}|f(x)|} \leq \frac{Q a(x)}{1+\frac{Q}{n} a(x)}=Q a_{n}(x) .
$$

We define:

$$
J(\omega)=\frac{1}{p} \hat{M}\left(\|\omega\|^{p}\right)+\int_{\Omega} a_{n}(x) g(v) \omega-\int_{\Omega} f_{n}(x) \omega,
$$

where $v \in W_{0}^{s, p}(\Omega)$, by $\mathrm{M}_{2}$ and (24), we can get

$$
J(\omega) \geq \frac{b}{p}\|\omega\|^{p \gamma}-\int_{\Omega} a_{n}(x)|g(v)-Q| \omega,
$$

by the Hölder's inequality and the Poincaré equality, then

$$
J(\omega) \geq \frac{b}{p}\|\omega\|^{p \gamma}-C a_{n}(x)\|g(v)-Q\|_{L^{q^{\prime}}}\|\omega\|,
$$

since $p \geq 2$ and $\gamma \in\left(1, p_{s}\right)$, then $J(\omega)$ is bounded, coercive and weakly lower semicontinuous, such that $J(\omega)$ has a minimizer and the Euler equation is:

$$
M\left(\|\omega\|^{p}\right) \Delta_{p}^{s} \omega+a_{n}(x) g(v)=f_{n} .
$$

Moreover, such a minimizer is unique, by the strict convexity of $J$.

Fixed $n \in N$, let $v \in W_{0}^{s, p}(\Omega)$, define $\omega=S(v)$ to be the unique solution of the problem:

$$
\begin{cases}M\left(\|\omega\|^{p}\right) \Delta_{p}^{s} \omega+a_{n}(x) g(v)=f_{n} & \text { in } \Omega, \\ \left.D^{\alpha} u(x)\right|_{\partial \Omega}=0 . & \forall \alpha, \text { with }|\alpha| \leq s-1 .\end{cases}
$$

We will use the $\omega$ as a test in (25), we get

$$
M\left(\|\omega\|^{p}\right) \int_{\Omega}\left|\mathcal{D}_{s} \omega\right|^{p}=\int_{\Omega} f_{n} \omega-\int_{\Omega} a_{n}(x) g(v) \omega,
$$

by $\left(M_{1}, M_{3}\right),(22),(24)$ and Hölder's inequality, we obtain

$$
m_{0}\|\omega\|^{p} \leq n\|\omega\|_{L^{1}}+\frac{n}{Q}\|g(v)\|_{L^{q^{q}}}\|\omega\|_{L^{q}} .
$$

where $q \in\left[1, p_{s}^{*}\right)$, and $q^{\prime}$ is the conjugate exponent of $q$, by (23) and the Poincaré equality, it follows that

$$
\|\omega\| \leq p-1 \frac{2 n C}{\frac{2 n}{m_{0}}}
$$

We take $R_{0}=\min \left\{\sqrt[p-1]{\frac{2 n C}{m_{0}}}, k_{0}\right\}$, so that the ball of radius $R_{0}>0$ is invariant 
under $s$ in $W_{0}^{s, p}(\Omega)$. In order to apply the Schauder's Fixed Point Theorem, apart from the invariant, we need to check the continuity and compactness of $s$ as an operator from $W_{0}^{s, p}(\Omega)$ to $W_{0}^{s, p}(\Omega)$. So, the proof will be divided into two steps.

Step 1: We prove the continuity. In order to do this, we define $\omega_{k}=S\left(v_{k}\right)$ and $\omega=S(v)$ then:

$$
\lim _{k \rightarrow \infty}\left\|\omega_{k}-\omega\right\|_{W_{0}^{s, p}(\Omega)}=0 \quad \text { where } \lim _{k \rightarrow \infty}\left\|v_{k}-v\right\|_{W_{0}^{s, p}(\Omega)}=0 .
$$

Since the convergence of $v_{k}$ in $W_{0}^{s, p}(\Omega)$, by (26) we obtain:

$$
\begin{aligned}
& v_{k} \rightarrow v \text { in } L^{q}(\Omega), \\
& v_{k} \rightarrow v \text { a.e in } \Omega, \quad q \in\left[1, p_{s}^{*}\right) .
\end{aligned}
$$

In fact, let $\left\{v_{k}\right\}_{k \in N}$, be a sequence in $W_{0}^{s, p}(\Omega)$ converging to $v \in W_{0}^{s, p}$.

To this end, by choosing $\omega_{k} \rightarrow \omega$ as a test function, we have

$$
\begin{gathered}
M\left(\left\|\omega_{k}\right\|^{p}\right) \int_{\Omega}\left|\mathcal{D}_{s} \omega_{k}\right|^{p-2} \mathcal{D}_{s}\left(\omega_{k}-\omega\right)=\int_{\Omega} f_{n}\left(\omega_{k}-\omega\right)-\int_{\Omega} a_{n}(x) g\left(v_{k}\right)\left(\omega_{k}-\omega\right), \\
M\left(\|\omega\|^{p}\right) \int_{\Omega}\left|\mathcal{D}_{s} \omega\right|^{p-2} \mathcal{D}_{s}\left(\omega_{k}-\omega\right)=\int_{\Omega} f_{n}\left(\omega_{k}-\omega\right)-\int_{\Omega} a_{n}(x) g(v)\left(\omega_{k}-\omega\right),
\end{gathered}
$$

by the inequality $\left(|x|^{p-2} x-|y|^{p-2} y\right)(x-y) \geq|x-y|^{p}$, for $x, y \in R$, and $p \geq 2$, by Hölder's inequality, we obtain

$$
\begin{aligned}
& m_{0}\left\|\omega_{k}-\omega\right\|^{p}+\left(M\left\|\omega_{k}\right\|^{p}-M\|\omega\|^{p}\right) \int_{\Omega}\left|\mathcal{D}_{s} \omega\right|^{p-2} \mathcal{D}_{s} \omega \mathcal{D}\left(\omega_{k}-\omega\right) \\
& \leq \frac{n}{Q}\left\|g\left(v_{k}\right)-g(v)\right\|_{L^{q}}\left\|\omega_{k}-\omega\right\|_{L^{q}}
\end{aligned}
$$

as $k \rightarrow \infty$,

$$
\begin{aligned}
& \left(M\left\|\omega_{k}\right\|^{p}-M\|\omega\|^{p}\right) \int_{\Omega}\left|\mathcal{D}_{s} \omega\right|^{p-2} \mathcal{D}_{s} \omega \mathcal{D}\left(\omega_{k}-\omega\right) \\
& \leq A \int_{\Omega}\left|\mathcal{D}_{s} \omega\right|^{p-2} \mathcal{D}_{s} \omega \mathcal{D}\left(\omega_{k}-\omega\right) \rightarrow 0
\end{aligned}
$$

where $A=b \gamma\left(\left\|\omega_{k}\right\|^{\gamma-1}+\|\omega\|^{\gamma-1}\right)$ is bounded, then by (27), and $g(v) \in L^{q^{\prime}}(\Omega)$, there is $\left\|\omega_{k}-\omega\right\| \rightarrow 0$, hence $S$ is continuous from $W_{0}^{s, p}(\Omega)$ to $W_{0}^{s, p}(\Omega)$.

Step 2. We prove $S$ is compact, first we take a sequence $\left\{v_{k}\right\}_{k \in N}$ that $\left\|v_{k}\right\| \leq C$, therefore by Rellich-Kondrachov Theorem, we obtain

$$
\begin{aligned}
& v_{k} \rightarrow v \text { in } W_{0}^{s, p}(\Omega) \\
& v_{k} \rightarrow v \text { in } L^{q}(\Omega), \quad 1 \leq q<p_{s}^{*}
\end{aligned}
$$

Since $S$ is continuous, $\left\|S\left(v_{k}\right)\right\| \leq C$.

with $S$ is a positive constant, independent of $k$, such that,

$$
\begin{aligned}
& S\left(v_{k}\right) \rightarrow \tilde{\omega} \text { in } W_{0}^{s, p}(\Omega) \\
& S\left(v_{k}\right) \rightarrow \tilde{\omega} \text { in } L^{q}(\Omega), \quad 1 \leq q<p_{s}^{*}
\end{aligned}
$$

Because of the continuity of $S$, necessarily $\tilde{\omega}=S(v)$, so that proceeding as in (28), we can get: 


$$
\begin{aligned}
& m_{0}\left\|S\left(v_{k}\right)-S(v)\right\|^{p}+\left(M\left\|S\left(v_{k}\right)\right\|^{p}-M\|S(v)\|^{p}\right) \\
& \times \int_{\Omega}\left|\mathcal{D}_{s} S(v)\right|^{p-2} \mathcal{D}_{s} S(v) \mathcal{D}\left(S\left(v_{k}\right)-S(v)\right) \\
& \leq \frac{n}{Q}\left\|g\left(v_{k}\right)-g(v)\right\|_{L^{q^{\prime}}}\left\|S\left(v_{k}\right)-S(v)\right\|_{L^{q}},
\end{aligned}
$$

as $k \rightarrow \infty$, the second term of left hand is vanished by (29) and (30). we can conclude

$$
\lim _{k \rightarrow \infty}\left\|S\left(v_{k}\right)-S(v)\right\|=0 .
$$

and therefore $S$ is compact.

Hence, by lemma 2.1, there exists a solution $u_{n} \in W_{0}^{s, p}(\Omega)$ of problem (21), next we will prove the problem (1).

We will use the following function defined for $t \in R$, by

$$
G_{k}(t)= \begin{cases}0 & \text { if }|t| \leq k \\ t-k & \text { if } t>k \\ t+k & \text { if } t<-k\end{cases}
$$

We use $G_{k_{0}}\left(u_{n}\right)$ as a test function in approximate problem (21), then

$$
M\left(\left\|u_{n}\right\|^{p}\right) \int_{\Omega}\left|\mathcal{D}_{s} G_{k_{0}}\left(u_{n}\right)\right|^{p}+\int_{\Omega} a_{n}(x) g\left(u_{n}\right) G_{k_{0}}\left(u_{n}\right)=\int_{\Omega} f_{n} G_{Q}\left(u_{n}\right)
$$

by $\left(\mathrm{M}_{1}, \mathrm{M}_{3}\right)$, and (24) we obtain:

$$
\begin{aligned}
& m_{0} \int_{\Omega}\left|\mathcal{D}_{s} G_{k_{0}}\left(u_{n}\right)\right|^{p}+\int_{\Omega} a_{n}(x) g\left(u_{n}\right) G_{k_{0}}\left(u_{n}\right) \\
& \leq \int_{\Omega} f_{n}\left|G_{Q}\left(u_{n}\right)\right| \leq Q \int_{\Omega} a_{n}(x)\left|G_{k_{0}}\left(u_{n}\right)\right|,
\end{aligned}
$$

by (9) and (23), this means:

$$
m_{0} \int_{\Omega}\left|\mathcal{D}_{s} G_{k_{0}}\left(u_{n}\right)\right|^{p}+\int_{\Omega} a_{n}(x)\left(\left|g\left(u_{n}\right)\right|-Q\right)\left|G_{k_{0}}\left(u_{n}\right)\right| \leq 0,
$$

which by (33) implies that $\left\|u_{n}\right\|_{L^{\infty}(\Omega)} \leq k_{0}$ and the sequence $\left\{u_{n}\right\}$ is bounded in $L^{\infty}(\Omega)$.

Next, we use $u_{n}$ as a test function to deduce, such that,

$$
M\left(\left\|u_{n}\right\|^{p}\right) \int_{\Omega}\left|\mathcal{D}_{s} u_{n}\right|^{p}+\int_{\Omega} a_{n}(x) g\left(u_{n}\right) u_{n}=\int_{\Omega} f_{n} u_{n}
$$

by $\left\|u_{n}\right\|_{L^{\infty}(\Omega)} \leq k_{0}$ and (23) we can get

$$
m_{0} \int_{\Omega}\left|\mathcal{D}_{s} u_{n}\right|^{p}-\max _{|t| \leq k_{0}}|g(t) t| \int_{\Omega} a_{n}(x) \leq k_{0} \int_{\Omega}\left|f_{n}\right|,
$$

then,

$$
m_{0} \int_{\Omega}\left|\mathcal{D}_{s} u_{n}\right|^{p} \leq \max _{|t| \leq k_{0}}|g(t) t| \int_{\Omega} a(x)+k_{0} \int_{\Omega}|f|,
$$

and we obtain that $\left\{u_{n}\right\}$ is bounded in $W_{0}^{s, p}(\Omega)$ and a sequence, still denoted $\left\{u_{n}\right\}$, which converges weakly in $W_{0}^{s, p}(\Omega)$ and a.e. to $u$ with $\|u\|_{L^{\infty}(\Omega)} \leq k_{0}$.

Moreover, using that $\left|a_{n}(x) g\left(v_{n}\right)\right| \leq a(x) \max _{|t| \leq k_{0}}|g(t)|$, we obtain by the dominated convergence theorem, the convergence of the sequence $\left\{a_{n}(x) g\left(v_{n}\right)\right\}$ to 
$\{a(x) g(u)\}$, which together with the $L^{1}(\Omega)$ convergence of $f_{n}(x)$ to $f(x)$, we pass to the limit in the problem (21), we prove that $u$ satisfies (1), with $u \in W_{0}^{s, p}(\Omega) \cap L^{\infty}(\Omega)$.

\section{A $p(x)$-polyharmonic Kirchhoff Equation}

In this section, we begin by recalling some basic results on the variable exponent Lebesgue and Sobolev spaces, see details in [22] [23].

As before, we define:

$$
C_{+}(\bar{\Omega})=\left\{h \in C(\bar{\Omega}): h_{-}>1\right\}
$$

where $\Omega \subset \mathbb{R}^{N}$ is a bounded domain, and $h_{+}=\max _{x \in \Omega} h(x), h_{-}=\max _{x \in \Omega} h(x)$. Let $h$ be the function in $C(\bar{\Omega})$, an important role in manipulating the generalized Lebesgue-Sobolev spaces is played by $L^{h(\cdot)}(\Omega)$ spaces, which is the convex function: $\rho_{h(\cdot)}: L^{h(\cdot)}(\Omega) \rightarrow R$ defined by:

$$
\rho_{h(\cdot)}(u)=\int_{\Omega}|u(x)|^{h(x)} \mathrm{d} x .
$$

Let $p$ be a fixed function in $C_{+}(\bar{\Omega})$. We endow the Luxemburg norm:

$$
\|u\|_{p(\cdot)}=\inf \left\{\lambda>0: \int_{\Omega}\left|\frac{u(x)}{\lambda}\right|^{p(x)} \mathrm{d} x \leq 1\right\} .
$$

by variable exponent Lebesgue space, it is a separable, reflexive Banach space. For $h \in C(\bar{\Omega}), \quad 1 \leq h \leq p$ in $\Omega$, then the embedding $L^{p(\cdot)}(\Omega) \hookrightarrow L^{h(\cdot)}(\Omega)$ is continuous and the norm of the embedding operator does not exceed $|\Omega|+1$. see [23].

$p^{\prime}$ be the function obtained by conjugating the exponent $p$ pointwise, so that $\frac{1}{p(x)}+\frac{1}{p^{\prime}(x)}=1$ for all $x \in \bar{\Omega}$, the $p^{\prime}$ belongs to $C_{+}(\bar{\Omega})$.

Note that, by Hölder-type inequality is valid:

$$
\int_{\Omega}|u(x) v(x)| \mathrm{d} x \leq C_{p(\cdot)}\|u\|_{p(\cdot)}\|v\|_{p^{\prime}(\cdot)} \quad \text { for all } u \in L^{p(\cdot)}(\Omega), v \in L^{p^{\prime}(\cdot)}(\Omega) .
$$

with $C_{p(\cdot)}=1+\frac{1}{p^{-}}-\frac{1}{p^{+}}$as proved in [23].

For $s=1,2, \cdots$, we introduced the variable exponent Sobolev space $W^{s, p(\cdot)}(\Omega)$ defined by:

$$
W^{s, p(\cdot)}(\Omega)=\left\{u \in L^{p(\cdot)}(\Omega): D^{\alpha} u \in L^{p(\cdot)}(\Omega) \text { for all } \alpha \in \mathbb{N}_{0}^{n}, \text { with }|\alpha| \leq s\right\},
$$

and endow the standard norm:

$$
\|u\|_{W^{s, p(\cdot)}(\Omega)}=\sum_{|\alpha| \leq s}\left\|D^{\alpha}\right\|_{p(\cdot)} .
$$

We point out that the nonstandard growth condition of $\left(p_{-}, p_{+}\right)$type.

Lemma 4.1. (Therorems 1.3 of [24]) If $\left(u_{k}\right)_{k}, u \in L^{h(\cdot)}(\Omega)$, with $1 \leq h_{-} \leq h_{+} \leq \infty$, then the following relations hold: 


$$
\left\{\begin{array}{l}
\|u\|_{h(\cdot)}<1(=1,>1) \Leftrightarrow \rho_{h(\cdot)}(u)<1(=1,>1) \\
\|u\|_{h(\cdot)} \geq 1 \Rightarrow\|u\|_{h(\cdot)}^{h_{-}} \leq\|u\|_{h(\cdot)} \leq\|u\|_{h(\cdot)}^{h_{+}} \\
\|u\|_{h(\cdot)} \leq 1 \Rightarrow\|u\|_{h(\cdot)}^{h_{+}} \leq\|u\|_{h(\cdot)} \leq\|u\|_{h(\cdot)}^{h_{-}}
\end{array}\right.
$$

and $\left\|u_{k}-u\right\| \rightarrow 0 \Leftrightarrow \rho_{h(\cdot)}\left(u_{k}-u\right) \rightarrow 0 \Leftrightarrow u_{k}-u$ in measure in $\Omega$ and $\rho_{h(\cdot)}\left(u_{k}\right) \rightarrow \rho_{h(\cdot)}(u)$. In particular, $\rho_{h(\cdot)}$ is continuous in $L^{h(\cdot)}(\Omega)$.

From now on we also assume that $p \in C_{+}^{\log }(\bar{\Omega})$, where $C_{+}^{\log }(\bar{\Omega})$ is the space of all the functions of $C_{+}(\bar{\Omega})$, which are logarithmic Hölder continuous, there exists $\mathcal{R}>0$, such that:

$$
|p(x)-p(y)| \leq-\frac{\mathcal{R}}{\log |x-y|}, \quad \text { for all } x, y \in \Omega .
$$

With $0<|x-y| \leq \frac{1}{2}$, the space $W_{0}^{s, p}(\Omega)$ denotes the completion of $C_{0}^{\infty}(\Omega)$ with respect to the norm $\|\cdot\|_{W^{s, p(\cdot)}(\Omega)}$.

Lemma 4.2. $W_{0}^{s, p(\cdot)}(\Omega)$ is a separable, uniformly convex, Banach space, see details in [22].

By the Poincaré inequality, see [25] [26], the equivalent norm for the space $W_{0}^{s, p(\cdot)}(\Omega)$ is given by:

$$
\|u\|_{\mathcal{D}^{s, p(\cdot)}(\Omega)}=\sum_{|\alpha|=s}\left\|\mathcal{D}^{\alpha} u\right\|_{p(\cdot)} .
$$

under this assumption, when $s=2$, as a consequence for the main ColdéronZygmund results, there exists a constant $k_{2}=k_{2}(n, p)>0$ such that:

$$
\|u\|_{\mathcal{D}^{2, p(\cdot)}(\Omega)} \leq k_{2}\|\Delta u\|_{p(\cdot)}=k_{2}\left\|\mathcal{D}_{2} u\right\|_{p(\cdot)} \quad \text { for all } u \in W_{0}^{s, p(\cdot)}(\Omega) .
$$

We recall that the operator $\mathcal{D}_{s}$ is defined in (4) is vectorial, when $s$ is odd, we endow $\left[L^{p(\cdot)}(\Omega)\right]^{n}$ space with the norm

$$
\|V\|_{p(\cdot)}=\sum_{i=1}^{n}\left\|\beta_{i}\right\|_{p(\cdot)} .
$$

where $V=\left(\beta_{1}, \beta_{2}, \cdots, \beta_{n}\right)$ with abuse of the notation we use the same symbol $\|\cdot\|_{p(\cdot)}$ to denote both the standard Luxemburg norm in the scalar space $L^{p(\cdot)}(\Omega)$ and the norm defined in (43) for the vectorial space $\left[L^{p(\cdot)}(\Omega)\right]^{n}$.

Proposition 4.1. See [17] for all $s=1,2, \cdots$ there exists $k_{s}=k_{s}(n, p)>0$, such that

$$
\|u\|_{\mathcal{D}^{s, p(\cdot)}(\Omega)} \leq k_{s}\left\|\mathcal{D}_{s} u\right\|_{p(\cdot)} \quad \text { for all } u \in W_{0}^{s, p(\cdot)}(\Omega)
$$

We endow the space $W_{0}^{s, p(\cdot)}$ with the norm $\|\cdot\|$, such that

$$
\|\cdot\|=\left\|\mathcal{D}_{s} \cdot\right\|_{p(\cdot)}
$$

Let $p_{s}^{*}$ denote the critical variable exponent related to $p$ defined for all $x \in \bar{\Omega}$, by the pointwise relation: 


$$
p_{s}^{*}(x)= \begin{cases}\frac{n p(x)}{n-s p(x)} & \text { if } n>s p(x) \\ \infty & \text { if } n \leq s p(x)\end{cases}
$$

If $s \geq n, p_{-}>1$ for all $h \in C(\bar{\Omega})$, the Sobolev embedding $W_{0}^{s, p(\cdot)}(\Omega) \hookrightarrow L^{h(\cdot)}(\Omega)$ is continuous and compact. If $p_{+}<\frac{n}{s}$ and $h \in C(\bar{\Omega})$, the embedding $W_{0}^{s, p(\cdot)}(\Omega) \hookrightarrow L^{h(\cdot)}(\Omega)$ is continuous whenever $1 \leq h(x) \leq p_{s}^{*}(x)$ for all $x \in \Omega$, there exists $\delta_{h^{+}}=\delta_{h^{+}}(n, p, s, \Omega)>0$ such that:

$$
\|u\|_{h(\cdot)} \leq \delta_{h^{+}}\|u\| \quad \text { for all } u \in W_{0}^{s, p(\cdot)}(\Omega)
$$

Moreover, for $1 \leq h_{-} \leq h_{+}<\left(p_{s}^{*}\right)_{-}$for all $x \in \Omega$ (or equivalent $1 \leq h(x)<p_{s}^{*}$ ), then $W_{0}^{s, p(\cdot)}(\Omega)$ is compactly embedded in $L^{h(\cdot)}$, see details in [17] [22] [24] [27].

Consider problem (10) with $s=1,2, \cdots$ and $p \in C_{+}^{\log }(\Omega)$, such that either $p_{+}<\frac{n}{S}$ or $p_{-} \geq \frac{n}{s}$. The Kirchhoff function $M: R_{0}^{+} \rightarrow R_{0}^{+}$is assumed to be continuous and to verify condition $(M)$ given in introduction, where $p_{s}=\frac{\left(p_{s}^{*}\right)_{-}}{p_{+}}$.

We denote the Dirchlet function $\varphi: W_{0}^{s, p(\cdot)}(\Omega) \rightarrow R_{0}^{+}$:

$$
\varphi(u)=\int_{\Omega} \frac{\left|\mathcal{D}_{s} u\right|^{p(x)}}{p(x)} \mathrm{d} x,
$$

where $\mathcal{D}_{s}$ is given by (4).

We study problem (10) for a solution we understand:

$$
\left\{\begin{array}{l}
u \in W_{0}^{s, p(\cdot)}(\Omega) \cap L^{\infty}(\Omega), \\
M(\varphi(u)) \int_{\Omega}\left|\mathcal{D}_{s} u\right|^{p(x)-2} \mathcal{D}_{s} u \mathcal{D}_{s} \varphi \mathrm{d} x+\int_{\Omega} a(x) g(u) \varphi=\int_{\Omega} f(x) \varphi \mathrm{d} x, \\
\varphi \in W_{0}^{s, p(\cdot)}(\Omega) \cap L^{\infty}(\Omega) .
\end{array}\right.
$$

where $\mathcal{D}_{s}$ is the operator in (4), and $\int_{\Omega}\left|\mathcal{D}_{s} u\right|^{p(x)-2} \mathcal{D}_{s} u \mathcal{D}_{s} \varphi \mathrm{d} x$ is the $p(x)$ -polyharmonic operator, $\Delta_{p(x)}^{s}$ in weak sense.

In order to study the solvability of problem (10), we will analyze the associated approximate problem.

$$
\begin{cases}M\left(\varphi\left(u_{n}\right)\right) \Delta_{p(x)}^{s} u_{n}+a_{n}(x) g\left(u_{n}\right)=f_{n} & \text { in } \Omega, \\ \left.D^{\alpha} u_{n}(x)\right|_{\partial \Omega}=0 & \forall \alpha, \text { with }|\alpha| \leq|s|-1 .\end{cases}
$$

We recall some basic conditions and hypothesis by (22)-(24), and exist $h(x) \in L^{p^{(\cdot)}}(\Omega)$, then $|g(s)| \leq h(x)$.

Theorem 4.1. There is a solution $u_{n} \in W_{0}^{s, p}(\Omega)$ to the problem (48).

Proof. We define

$$
J(\omega)=\hat{M}(\varphi(\omega))+\int_{\Omega} a_{n}(x) g(v) \omega-\int_{\Omega} f_{n} \omega .
$$


Observe that, by (46), and lemma 4.1 we get:

$$
\varphi(\omega)=\int_{\Omega} \frac{\left|\mathcal{D}_{s} \omega\right|^{p(x)}}{p(x)} \mathrm{d} x \geq \frac{1}{p_{+}} \rho_{p(\cdot)}\left(\mathcal{D}_{s} \omega\right) \geq \frac{1}{p_{+}} \min \left\{\|\omega\|^{p_{+}},\|\omega\|^{p_{-}}\right\} .
$$

we take $\delta=\frac{1}{p_{+}} \min \left\{\|\omega\|^{p_{+}},\|\omega\|^{p_{-}}\right\}>0$, so that by $\left(\mathrm{M}_{1}, \mathrm{M}_{3}\right)$, there exists $m_{0}(\delta)$,

$$
M(\varphi(\omega)) \geq m_{0} .
$$

by $M_{2}$ and (49), we can get:

$$
J(\omega) \geq b \delta^{\gamma}-\int_{\Omega} a_{n}(x)|g(v)-Q| \omega .
$$

by the Hölder's inequality and the Poincaré equality, then

$$
J(\omega) \geq b \delta^{\gamma}-C a_{n}(x)\|g(v)-Q\|_{L^{p^{\prime}(\cdot)}}\|\omega\|,
$$

since $a(x) \geq 0$ and $g(v) \in L^{p^{\prime}(\cdot)}(\Omega)$, then $J(\omega)$ is bounded, coercive and weakly lower semicontuous, such that $J(\omega)$ has a minimizer and the Euler equation is

$$
M(\varphi(\omega)) \Delta_{p(x)}^{s} \omega+a_{n}(x) g(v)=f_{n} .
$$

Moreover, such a minimizer is unique, by the strict convexity of $J$.

Fix $n \in N$, let $v \in W_{0}^{s, p(\cdot)}(\Omega)$, define $\omega=s(v)$ to be the unique solution of the problem:

$$
\begin{cases}M(\varphi(\omega)) \Delta_{p(x)}^{s} \omega=f_{n}-a_{n}(x) g(v) & \text { in } \Omega \\ \left.D^{\alpha} u(x)\right|_{\partial \Omega}=0 . & \forall \alpha, \text { with }|\alpha| \leq s-1\end{cases}
$$

We will use the $\omega$ as a test in (51) we get:

$$
M(\varphi(\omega)) \int_{\Omega}\left|\mathcal{D}_{s} \omega\right|^{p(x)}=\int_{\Omega} f_{n} \omega-\int_{\Omega} a_{n}(x) g(v) \omega,
$$

by (22), (24), (50) and Hölder's inequality, thus,

$$
m_{0} \rho_{p(\cdot)}\left(\mathcal{D}_{s} \omega\right) \leq n\|\omega\|_{L^{1}}+C_{p(\cdot)} \frac{n}{Q}\|g(v)\|_{p^{\prime}(\cdot)}\|\omega\|_{p(\cdot)} .
$$

1) If $\|\omega\| \geq 1$, such that

$$
\begin{gathered}
m_{0}\|\omega\|^{p_{-}} \leq n C_{1}\|\omega\|+C_{p(\cdot)} n C_{2}\|\omega\|, \\
\|\omega\| \leq \sqrt[p_{-}-1]{\frac{n\left(C_{1}+C_{p(\cdot)} C_{2}\right)}{m_{0}}} .
\end{gathered}
$$

2) If $\|\omega\| \leq 1$, such that

$$
\begin{gathered}
m_{0}\|\omega\|^{p_{+}} \leq n C_{1}\|\omega\|+C_{p(\cdot)} n C_{2}\|\omega\|, \\
\|\omega\| \leq \sqrt[p_{+}-1]{\frac{n\left(C_{1}+C_{p(\cdot)} C_{2}\right)}{m_{0}}} .
\end{gathered}
$$


We take $R_{0}=\min \left\{\sqrt[p_{-}-1]{\frac{n\left(C_{1}+C_{p(\cdot)} C_{2}\right)}{m_{0}}}, \sqrt[p_{+}-1]{\frac{n\left(C_{1}+C_{p(\cdot)} C_{2}\right)}{m_{0}}}, k_{0}\right\}$, so that the ball of radius $R_{0}>0$ is invariant under $s$ in $W_{0}^{s, p(\cdot)}(\Omega)$. In order to apply the Schauder's Fixed Point Theorem, apart from the invariant, we need to check the continuity and compactness of $s$ as an operator from $W_{0}^{s, p(\cdot)}(\Omega)$ to $W_{0}^{s, p(\cdot)}(\Omega)$. So, the proof will be divided into two steps.

Step 1: We prove the continuity. In order to do this, we define $\omega_{k}=S\left(v_{k}\right)$ and $\omega=S(v)$ then:

$$
\lim _{k \rightarrow \infty}\left\|\omega_{k}-\omega\right\|_{W_{0}^{s, p(\cdot)}(\Omega)}=0 \quad \text { where } \lim _{k \rightarrow \infty}\left\|v_{k}-v\right\|_{W_{0}^{s, p}(\Omega)}=0 .
$$

Since the convergence of $v_{k}$ in $W_{0}^{s, p(\cdot)}(\Omega)$, by (52) we obtain:

$$
v_{k} \rightarrow v \text { in } L^{h(\cdot)}(\Omega), v_{k} \rightarrow \text { va.e in } \Omega \quad h(\cdot) \in\left[1, p_{s}^{*}\right) .
$$

In fact, let $\left\{v_{k}\right\}_{k \in N}$, be a sequence in $W_{0}^{s, p(\cdot)}(\Omega)$ converging to $v \in W_{0}^{s, p(\cdot)}$.

To this end, by choosing $\omega_{k} \rightarrow \omega$ as a test function, we have

$$
\begin{aligned}
& M\left(\varphi\left(\omega_{k}\right)\right) \int_{\Omega}\left|\mathcal{D}_{s} \omega_{k}\right|^{p(x)-2} \mathcal{D}_{s}\left(\omega_{k}-\omega\right) \\
& =\int_{\Omega} f_{n}\left(\omega_{k}-\omega\right)-\int_{\Omega} a_{n}(x) g\left(v_{k}\right)\left(\omega_{k}-\omega\right), \\
& M(\varphi(\omega)) \int_{\Omega}\left|\mathcal{D}_{s} \omega\right|^{p(x)-2} \mathcal{D}_{s}\left(\omega_{k}-\omega\right) \\
& =\int_{\Omega} f_{n}\left(\omega_{k}-\omega\right)-\int_{\Omega} a_{n}(x) g\left(v_{k}\right)\left(\omega_{k}-\omega\right),
\end{aligned}
$$

by the inequality $\left(|x|^{p-2} x-|y|^{p-2} y\right)(x-y) \geq|x-y|^{p}$ for $x, y \in R, \quad p \geq 2$, (22), (38) and Hölder's inequality, we obtain:

$$
\begin{aligned}
& m_{0} \rho_{p(\cdot)}\left(\mathcal{D}_{s}\left(\omega_{k}-\omega\right)\right)+\left(M\left(\varphi\left(\omega_{k}\right)\right)-M(\varphi(\omega))\right) \int_{\Omega}\left|\mathcal{D}_{s} \omega\right|^{p(x)-2} \mathcal{D}_{s} \omega \mathcal{D}_{s}\left(\omega_{k}-\omega\right) \\
& \leq C_{p(\cdot)} \frac{n}{Q}\left\|g\left(v_{k}\right)-g(v)\right\|_{p^{\prime}(\cdot)}\left\|\omega_{k}-\omega\right\|_{p(\cdot)}
\end{aligned}
$$

as $k \rightarrow \infty$,

$$
\left(M\left(\varphi\left(\omega_{k}\right)\right)-M(\varphi(\omega))\right) \leq \frac{1}{p_{-}} A \int_{\Omega}\left|\mathcal{D}_{s} \omega\right|^{p(x)-2} \mathcal{D}_{s} \omega \mathcal{D}_{s}\left(\omega_{k}-\omega\right) \rightarrow 0 .
$$

where $A=\max \left\{\left\|\omega_{k}\right\|^{p_{-}},\left\|\omega_{k}\right\|^{p_{+}}\right\}+\max \left\{\|\omega\|^{p_{-}},\|\omega\|^{p_{+}}\right\}$is bounded, since (53) and $g$ is continuous, such that

$$
\rho_{p(\cdot)}\left(\mathcal{D}\left(\omega_{k}-\omega\right)\right) \rightarrow 0 \Rightarrow\left\|\omega_{k}-\omega\right\| \rightarrow 0,
$$

hence $s$ is continuous from $W_{0}^{s, p(\cdot)}(\Omega)$ to $W_{0}^{s, p(\cdot)}(\Omega)$.

Step 2. We prove $S$ is compact, first we take a sequence $\left\{v_{k}\right\}_{k \in N}$ that $\left\|v_{k}\right\| \leq C$, therefore by Rellich-Kondrachov Theorem, we obtain

$$
\begin{aligned}
& v_{k} \rightarrow v \text { in } W_{0}^{s, p(\cdot)}(\Omega) \\
& v_{k} \rightarrow v \text { in } L^{h(\cdot)}(\Omega), \quad 1 \leq h(\cdot)<p_{s}^{*}
\end{aligned}
$$

Since $S$ is continuous, $\left\|S\left(v_{k}\right)\right\| \leq C$.

with $C$ is a positive constant, independent of $k$, such that, 


$$
\begin{aligned}
& S\left(v_{k}\right) \rightarrow \tilde{\omega} \text { in } W_{0}^{s, p(\cdot)}(\Omega) \\
& S\left(v_{k}\right) \rightarrow \tilde{\omega} \text { in } L^{h(\cdot)}(\Omega), \quad 1 \leq h(\cdot)<p_{s}^{*}
\end{aligned}
$$

Because of the continuity of $S$, necessarily $\tilde{\omega}=S(v)$, so that proceeding as in (54), we can get

$$
\begin{aligned}
& \left(M\left(\varphi\left(S\left(v_{k}\right)\right)\right)-M(\varphi(S(v)))\right) \int_{\Omega}\left|\mathcal{D}_{s} S(v)\right|^{p(x)-2} \mathcal{D}_{s} S(v) \mathcal{D}_{s}\left(S\left(v_{k}\right)-S(v)\right) \\
& +m_{0} \rho_{p(\cdot)} \mathcal{D}_{s}\left(S\left(v_{k}\right)-S(v)\right) \\
& \leq C_{p(\cdot)} \frac{n}{Q}\left\|g\left(v_{k}\right)-g(v)\right\|_{p^{\prime}(\cdot)}\left\|S\left(v_{k}\right)-S(v)\right\|_{p(\cdot)},
\end{aligned}
$$

as $k \rightarrow \infty$, the first term of the left hand is vanished, then by (55) and (56)

$$
\rho_{p(\cdot)} \mathcal{D}\left(S\left(v_{k}\right)-S(v)\right) \rightarrow 0 \Rightarrow\left\|S\left(v_{k}\right)-S(v)\right\| \rightarrow 0,
$$

therefore, $S$ is compact.

Given these conditions on $S$, Schauder's Fixed Point Theorem provides the existence of $u_{n} \in W_{0}^{s, p(\cdot)}(\Omega)$, such that $u_{n}=S\left(u_{n}\right)$, i.e., $u_{n}$ solves:

$$
\begin{cases}M\left(\varphi\left(u_{n}\right)\right) \Delta_{p(x)}^{s} u_{n}+a_{n}(x) g\left(u_{n}\right)=f_{n} & \text { in } \Omega, \\ \left.D^{\alpha} u_{n}(x)\right|_{\partial \Omega}=0 & \forall \alpha, \text { with }|\alpha| \leq|s|-1 .\end{cases}
$$

By Section 3, we also use $G_{k}\left(u_{n}\right)$ as a test function, then we can obtain the sequence $\left\{u_{n}\right\}$ is bounded in $L^{\infty}(\Omega)$, next we will use $u_{n}$ as a test function, we can get a sequence $u_{n}$, which converges weakly in $W_{0}^{s, p(\cdot)}(\Omega)$ and a.e. to $u$ with $\|u\|_{L^{\infty}} \leq k_{0}$. Finally, by the dominated convergence theorem and $L^{1}$ convergence, we prove that $u$ satisfies (10) with $u \in W_{0}^{s, p(\cdot)}(\Omega) \cap L^{\infty}(\Omega)$.

Thus, we can learn some Kirchhoff equations by the above method.

\section{Acknowledgements}

We closely thank the following instructions. It will definitely save a lot of time and expedite the process of your paper's publication.

\section{Conflicts of Interest}

The author declares no conflicts of interest regarding the publication of this paper.

\section{References}

[1] Kratochvil, A. and Necas, J. (1971) The Discreteness of the Spectrum of a Nonlinear Strum-Liouville Equation of Fourth Order. Commentationes Mathematicae Universitatis Carolinae, 12, 639-653.

[2] Drabek, P. and Otani, M. (2001) Global Bifurcation Result for the p-Biharmonic Operator. Electronic Journal of Differential Equations, 2001, 1-19.

[3] El Khalil, A., Kellati, S. and Touzani, A. (2002) On the Spectrum of the p-Biharmonic Operator. Electronic Journal of Differential Equations, 9, 161-170.

[4] Correa, F.I.S.A. and Fijueiredo, G.M. (2006) On an Elliptic Equation of $p$-Kirchhoff Type via Variational Methods. Bulletin of the Australian Mathematical Society 74, 
236-277. https://doi.org/10.1017/S000497270003570X

[5] Ma, T.F. (2005) Remarks on an Elliptic Equation of Kirchhoff Type. Nonlinear Analysis, 63, 1967-1977. https://doi.org/10.1016/j.na.2005.03.021

[6] Ma, T.F. (2005) Positive Solutions for a Nonlinear Kirchhoff Type Beam Equation. Applied Mathematics Letters, 18, 479-482. https://doi.org/10.1016/j.aml.2004.03.013

[7] Autuori, G., Pucci, P. and Salvatori, M.C. (2009) Asymptotic Stability for Nonlinear Kirchhoff Systems. Nonlinear Analysis, 10, 889-809.

https://doi.org/10.1016/j.nonrwa.2007.11.011

[8] Villaggio, P. 1997 Mathmatical Models for Elastic Structures. Cambridge University Press, Cambridge. https://doi.org/10.1017/CBO9780511529665

[9] Cavalcanti, M.M., Domingos Cavalcanti, V.N. and Soriano, J.A. (2001) Global Existence and Uniform Decay Rates for the Kiechhoff-Carrier Equation with Nonlinear Dissipation. Advances in Differential Equations, 6, 701-730.

[10] D’Ancona, P. and Spagnolo, S. (1992) Global Solvability for the Degenerate Kirchhoff Equation with Real Analytic Data. Inventiones Mathematicae, 108, 247-262. https://doi.org/10.1007/BF02100605

[11] Dai, G. and Hao, R. (2009) Existence of Solutions for a $p(x)$-Kirchhoff-Type Equation. Journal of Mathematical Analysis and Applications, 359, 275-284. https://doi.org/10.1016/j.jmaa.2009.05.031

[12] Dai, G. and Wei, J. (2010) Infinitely Many Non-Negative Solutions for a $p(x)$ -Kirchhoff-Type Problem with Dirchlet Boundary Condition. Nonlinear Analysis: Theory, Methods \& Applications, 73, 3420-3430. https://doi.org/10.1016/j.na.2010.07.029

[13] Benilan, P., Brezis, H. and Crandall, M.G. (1975) A Semilinear Equation in $L^{1}\left(\mathbb{R}^{N}\right)$. Annali della Scuola Normale Superiore di Pisa, 2, 523-555.

[14] Boccardo, L., Murat, F. and Puel, J.P. (1992) $L^{\infty}$-Estimate for Nonlinear Elliptic Partial Differential Equations and Application to an Existence Result. SIAM Journal on Mathematical Analysis, 23, 326-333. https://doi.org/10.1137/0523016

[15] Arcoya, D. and Boccardo, L. (2015) Regularizing Effect of the Interplay between Coefficients in Some Epllitic Equations. Journal of Functional Analysis, 268, 1153-1166. https://doi.org/10.1016/j.jfa.2014.11.011

[16] Arcoya, D. and Boccardo, L. (2017) Regularizing Effect of $L^{q}$ Interplay between Coefficients in Some Epllitic Equations. Journal de Mathématiques Pures et Appliquées, 111, 106-125. https://doi.org/10.1016/j.matpur.2017.08.001

[17] Colasuonno, F. and Pucci, P. (2011) Multiplicity of Solutions for $p(x)$-Polyharmonic Elliptic Kirchhoff Equations. Nonlinear Analysis, 74, 5962-5974.

https://doi.org/10.1016/j.na.2011.05.073

[18] Adams, R.A. and Fournier, J.J.F. (2003) Sobolev Spaces. In: Pure and Applied Mathematics, 2nd Edition, Springer, Amsterdam.

[19] Gilbarg, D. and Trudinger, N. (2001) Elliptic Partial Differential Equations of Second Order. In: Classics in Mathematics, Springer, Berlin.

[20] Gazzola, F., Grunau, H.C. and Sweers, G. (2010) Polyharmonic Boundary Problems. Positivity Preserving and Nonlinear Higher Order Elliptic Equations in Bounded Domains. In: Lecture Notes in Mathematics, Springer, Berlin. https://doi.org/10.1007/978-3-642-12245-3

[21] Boccardo, L. and Croce, G. (2013) Elliptic Partial Differential Equations (Existence 
and Regularity of Distributional Solutions). De Gruyter, Berlin. https://doi.org/10.1515/9783110315424

[22] Dinening, L., Harjulehto, P., Hasto, P. and Ruzicka, M. (2011) Lebesgue and Sobolev Speaces with Variable Exponents. In: Lecture Notes, Springer, Berlin. https://doi.org/10.1007/978-3-642-18363-8

[23] Kovacik, O. and Rakosnik, J. (1991) On Spaces $L^{p(x)}$ and $W^{1, p(x)}$. Czechoslovak Mathematical Journal, 41, 592-618.

[24] Fan, X.L. and Zhao, D. (2001) On the Spaces $L^{p(x)}$ and $W^{m, p(x)}$. Journal of Mathematical Analysis and Applications, 263, 424-446. https://doi.org/10.1006/jmaa.2000.7617

[25] Harjulehto, P., Hasto, P., Koskenoja, M. and Varonen, S. (2006) The Dirchlet Energy Integral and Variable Exponents Sobolev Speaces with Zero Boundary Values, Potential Analysis, 25, 205-222. https://doi.org/10.1007/s11118-006-9023-3

[26] Diening, L. and Ruzicka, M. (2003) Calderon-Zygmund Operators on Generalized Lebesgue Spaces $L^{p(\cdot)}$ and Problems Related to Fluid Dynamics. Journal für die reine und angewandte Mathematik, 563, 197-220. https://doi.org/10.1515/crll.2003.081

[27] Dinening, L. (2004) Riesz Potential and Sobolev Embeddings on Generalized Lebesdue and Sobolev Speaces $L^{p(\cdot)}$ and $W^{k, p(\cdot)}$. Mathematische Nachrichten, 268, 31-43. https://doi.org/10.1002/mana.200310157 